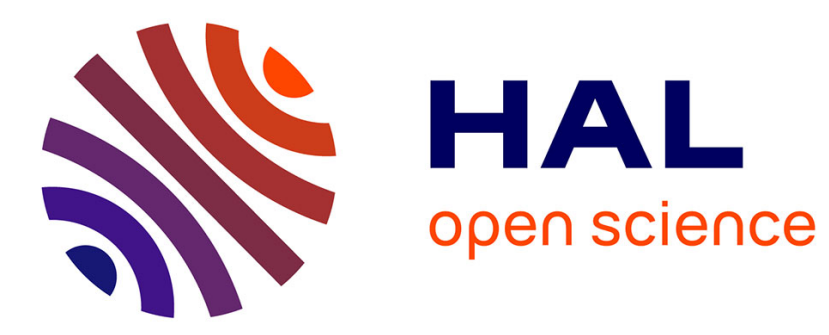

\title{
Affine Stereo Calibration
}

\author{
Peter Sturm, Long Quan
}

\section{To cite this version:}

Peter Sturm, Long Quan. Affine Stereo Calibration. 6th International Conference on Computer Analysis of Images and Patterns (CAIP '95), Sep 1995, Prague, Czech Republic. pp.838-843, 10.1007/3540-60268-2_390. inria-00525706

\section{HAL Id: inria-00525706 https://hal.inria.fr/inria-00525706}

Submitted on 31 May 2011

HAL is a multi-disciplinary open access archive for the deposit and dissemination of scientific research documents, whether they are published or not. The documents may come from teaching and research institutions in France or abroad, or from public or private research centers.
L'archive ouverte pluridisciplinaire HAL, est destinée au dépôt et à la diffusion de documents scientifiques de niveau recherche, publiés ou non, émanant des établissements d'enseignement et de recherche français ou étrangers, des laboratoires publics ou privés. 


\title{
Affine stereo calibration
}

\author{
Peter Sturm and Long Quan \\ Lifia-Inria, 46, Avenue Félix-Viallet, 38031 Grenoble, France \\ Peter.Sturm@inrialpes.fr • http://www.inrialpes.fr/movi/people/Sturm/
}

\begin{abstract}
Affine stereo calibration has been identified as the determination of the plane collineation induced by the plane at infinity. Generally, this so-called infinity homography can be determined provided 3 image correspondences of points at infinity. In this paper, we first show that affine stereo calibration for the case of two cameras with the same intrinsic parameters is possible with one less point at infinity. Then, we propose two practical methods for affine calibration in the general case. The experimental results on simulated and real images are presented.
\end{abstract}

\section{Introduction}

A basic task in computer vision applications is the determination of camera parameters, the camera calibration. Camera calibration is the basis of how to obtain 3D reconstructions which can be used to recognize objects, navigate in an unknown environment, or just to give binary informations about the scene, like "there is/is not an obstacle in front of the cameras". It is evident that, for all 3 examples, exact $3 \mathrm{D}$ reconstruction is not always necessary. Object recognition can be realized on the basis of Euclidean as well as affine or projective invariants. Navigation sub-tasks often require knowledge of only affine instead of Euclidean scene properties. For simple obstacle detection, the identification of a plane in the scene and the epipolar geometry of the camera system are sufficient.

In the pioneer work on projective reconstruction from two uncalibrated images $[2,6]$, the affine reconstruction proposed was determined up to 3 free parameters. This is later clarified in [9] in which it was shown that the affine calibration is equivalent to determine the infinity homography.

The goals of this paper are twofold. Firstly, we explore the theory of affine calibration for cameras with same intrinsic parameters, or, equivalently, for one camera in movement. Secondly we emphasize practical aspects of affine calibration which lead to the proposition of two practical methods of affine calibration.

\section{Preliminaries}

\subsection{Camera model}

The camera model used throughout the paper is the pin-hole model which models the camera projection as a perspective transformation from $\mathcal{P}^{3}$ to $\mathcal{P}^{2}$, represented by a matrix $P$. This projection matrix can be decomposed as

$$
P_{3 \times 4}=K D=K\left(\begin{array}{lll}
R & \mid-R \mathbf{t}
\end{array}\right)=\left(\begin{array}{lll}
K R & \mid-K R \mathbf{t}
\end{array}\right) .
$$


$R$ and $\mathbf{t}$ represent the extrinsic and $\mathrm{K}$ the intrinsic parameters of the camera.

\subsection{Projective calibration}

Projective or weak calibration consists on the determination of the epipolar geometry of two cameras $[2,3,6]$. Projective calibration enables the determination of the projection matrices of the two cameras, up to an unknown, but common, projective transformation [5].

\subsection{Affine calibration}

Knowing the epipolar geometry and the plane collineation for the plane at infinity, affine reconstruction is possible [9]. We call therefore affine calibration the determination of the so-called infinity homography, provided a projective calibration is given. The infinity homography is represented by the $3 \times 3$ matrix $H_{\infty}$, which can be computed by $[9]^{1}$

$$
H_{\infty} \approx K^{\prime} R^{\prime}(K R)^{-1}=K^{\prime} R^{\prime} R^{T} K^{-1} .
$$

It can be determined from 3 correspondences of vanishing points, provided the epipolar geometry is given (the two epipoles give a fourth point correspondence).

\section{Affine calibration of two cameras with same intrinsic parameters}

\subsection{A constraint for the infinity homography}

For two cameras with same intrinsic parameters, i.e. $K^{\prime}=K$, Equation (1) is simplified:

$$
H_{\infty} \approx K R^{\prime}(K R)^{-1}=K R^{\prime} R^{T} K^{-1}=K R^{\prime \prime} K^{-1}
$$

where $R^{\prime \prime}$ is a rotation matrix. Hence, $H_{\infty}$ is similar to a multiple ${ }^{2}$ of $R^{\prime \prime}$ and has eigenvalues $\lambda, \lambda e^{i \theta}$, and $\lambda e^{-i \theta}$, with a $\lambda \in \mathcal{R}$. Thus, the eigenvalues of $H_{\infty}$ have the same module. This constraint should be taken into account in the estimation of $H_{\infty}$ when the two cameras have same intrinsic parameters [7].

\subsection{Determination of the infinity homography}

A constructive proof of the following proposition is given in [10].

Proposition 1. Suppose that two cameras have same intrinsic parameters and the epipolar geometry for the cameras is known. Suppose further that the correspondences of 2 vanishing points are known.

Then, in non-degenerated cases (made precise in the proof), we can determine the infinity homography up to at most 6 solutions.

\footnotetext{
${ }^{1} \mathrm{By} \approx$ we mean equality up to scalar multiplication.

${ }^{2}$ Multiple of a matrix means here the matrix, multiplied with any scalar.
} 


\section{Practical affine calibration methods}

We propose two practical methods of affine calibration in the general case, i.e. the cameras need not to have the same intrinsic parameters. Both of the methods suppose that a projective calibration is given. Their principle is to determine the infinity homography by correspondences of vanishing points or lines.

We make the assumption that the stereo system undergoes a translational movement relative to the observed scene.

Detection of vanishing points. Scene points move on parallel trajectories, relative to the stereo system. In each image, the vanishing point for the translational direction is just the intersection of the straight lines which join the tracked images of one scene point.

Detection of vanishing lines. Consider some coplanar scene points which span a plane $\Pi$. As viewed from the stereo system, $\Pi$ undergoes a translational movement. Let $\Pi_{i}$ be the translated plane at the $i$-th instant. Since all the $\Pi_{i}$ are parallel, they intersect in a line at infinity. We briefly describe how the projections of this line, its vanishing lines, can be determined. For every instant $i$ where tracking of the points on $\Pi$ is done, we carry out a projective reconstruction of $\Pi_{i}$, using the projective calibration already given. To do so, we reconstruct the points lying on $\Pi_{i}[8,4]$ and obtain the plane in the projective space by spanning the reconstructed points. We intersect the planes and back-project the intersection line into the image planes, using once again the given projective calibration.

First affine calibration method: Observation of a plane during a translational movement. We consider a translational movement between the stereo system and the scene. In order to be able to detect the vanishing points for the translational direction, tracking of 2 scene points is sufficient. Detection of a vanishing line requires tracking of at least 3 coplanar points. Since 3 points are always coplanar, tracking of at least 3 scene points is sufficient to determine one correspondence of vanishing points and one of vanishing lines. Once these correspondences have been established, we can estimate the infinity homography.

The extension of the method for more than 3 points is straightforward.

Second affine calibration method: Three translational movements. With a translational movement between the stereo system and the scene we can determine one correspondence of vanishing points. Thus, with 3 different translational movements, which are not in the same plane, we have enough corrspondences of vanishing points to determine the infinity homography.

The extension of the method for more than 3 movements is straightforward. 


\section{Experimental results}

\subsection{Outline of the experiments}

We undertook experiments with simulated as well as real image data for the first of the two described affine calibration methods. The different steps of the experimentation are:

1. Determination of the epipolar geometry using an iterative algorithm.

2. Affine calibration of the stereo system by observation of a plane during translational movement.

3. Euclidean calibration with a classical model-based method.

4. Affine reconstruction of the scene.

5. Euclidean reconstruction of the same scene. In experimentation with simulated data we take here the original 3D point set.

6. Evaluating the quality of affine calibration by comparing the affine reconstruction with the Euclidean one (see 5.2).

\subsection{Evaluation of the quality of affine reconstruction}

Suppose that we have reconstructed $n$ points, where the $Q_{i}$ are the affine reconstruction and the $R_{i}$ the Euclidean reconstruction. We evaluate the quality of the affine reconstruction by the following value:

$$
\frac{1}{n} \min _{T \in \mathcal{A}_{3}} \sum_{i=1}^{n}\left|R_{i}-T Q_{i}\right|
$$

where $\mathcal{A}_{3}$ is the group of affine transformations on $\mathcal{P}^{3}$. In words: we align the affine reconstruction with the Euclidean one. Then, the mean distance error serves as quality value of the affine reconstruction.

\subsection{Experiments with simulated data}

We have done affine calibration for all pairs of 9 virtual cameras, by observing a plane with 92 points which has been translated 4 times. 60 object points have then been projected by the cameras and the image coordinates perturbed by Gaussian or uniform noise from 1 to 5 pixel. The affine reconstruction, done for each pair of the 9 cameras, has then been compared with the original point set, using the criterion defined in 5.2.

Table 1 shows the obtained results where each of the error values is the mean of 36 different experiments with 60 object points. The error is given in affine unit which can be related to the size of the object, $30 \times 41 \times 22.5$. 


\begin{tabular}{|l|c|c|c|r|c|c|c|c|c|c|}
\hline & \multicolumn{4}{|c|}{ Gaussian noise } & \multicolumn{4}{c|}{ Uniform noise } \\
\hline Noise level & 1 & 2 & 3 & 4 & 5 & 1 & 2 & 3 & 4 & 5 \\
\hline Error & 0.329 & 1.468 & 2.942 & 8.878 & 7.421 & 0.128 & 0.116 & 0.152 & 0.207 & 0.266 \\
\hline Variance & 0.053 & 10.894 & 13.834 & 112.268 & 40.258 & 0.105 & 0.007 & 0.007 & 0.017 & 0.028 \\
\hline
\end{tabular}

Table 1. The results of experiments with simulated data.

\subsection{Experiments with real data}

The image points used for affine calibration have been obtained by taking 5 pairs of images of a calibration grid which was subject to a translational movement between each taking, but always in the same direction. The images of the calibration grid have been independently used to establish a Euclidean calibration of the stereo system. Some sample scenes for experiments are shown in Table 2.

The values in Table 2 show the errors of the affine reconstructions in comparison to the Euclidean ones (see Section 5.2). Each value is the mean of 8 different calibration setups. In order to well interpret the error values it is important to note that the height of the house is about $30 \mathrm{~cm}$.

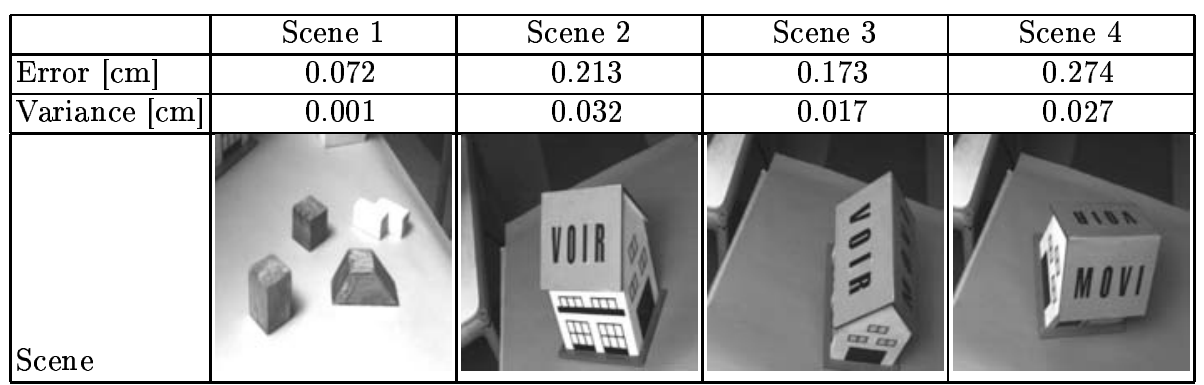

Table 2. The results of experiments with real images. Errors and variances are given in $\mathrm{cm}$.

Another important remark is that the images have been taken at distances inferior to $1.5 \mathrm{~m}$. So, $\frac{\text { distance object-camera }}{\text { object's size }}<5$. Normally, under this condition, the assumption of an affine camera model would give bad results. Since we are using an entirely projective model, our affine calibration method has no trouble with this condition.

In Figure 1 two examples of affine reconstructions of scenes 1 and 2 are shown, in comparison with Euclidean reconstructions.

\section{Discussion}

In this paper, we have examined affine calibration for cameras with same intrinsic parameters and have shown that in this case only 2 instead of 3 correspondences of vanishing points are needed. 


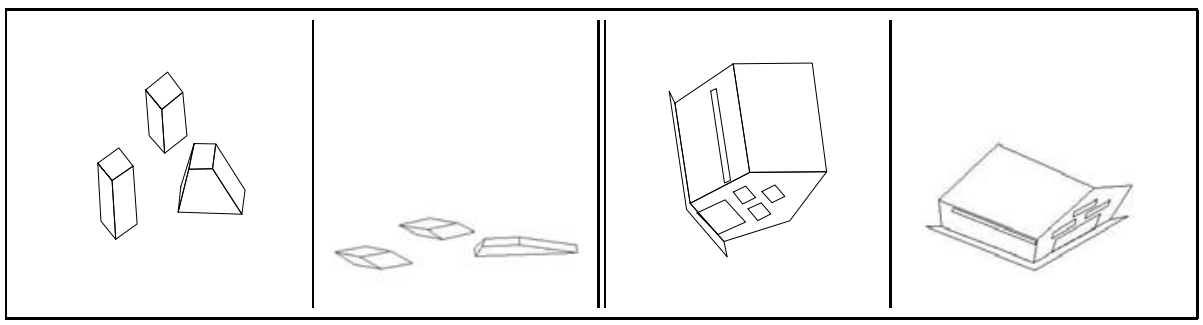

Fig. 1. Euclidean and affine reconstructions of two scenes.

We have proposed two practical methods of general affine stereo calibration which can directly be applied for affine self-calibration. Only for the calibration step a translational movement of the camera system would be necessary.

Our approach goes in the same direction as the one proposed in [1] where the camera system is supposed to move only in a single plane and to rotate around axes which are perpendicular to this plane. With that approach, which is also well applicable for vehicles, 3 stereo pairs are needed for affine calibration whereas our approach needs only 2 . This is due to our assumption of a translational movement which is a little bit stronger than the one used in [1].

We have experimented one of the proposed affine calibration methods with simulated as well as real data and the results show the applicability of the approach.

This work has been partly supported by ESPRIT-BRA No. 6769 "Second", which is grateful acknowledged.

\section{References}

1. Beardsley, P.A., Zisserman, A.: Affine Calibration of Mobile Vehicles. Proc. of the Workshop on Geometrical modelling and invariant computer vision, China, 1995

2. Faugeras, O.: What can be seen in three dimensions with an uncalibrated stereo rig?. Proc. of the 2nd ECCV, Santa Margherita Ligure, Italy, 1992, 563-578

3. Faugeras, O.D., Luong Q.T., Maybank S.J.: Camera Self-Calibration: Theory and Experiments. Proc. of the 2nd ECCV, Santa Margherita Ligure, Italy, 1992, 321-334

4. Hartley, R., Sturm, P.: Triangulation. Proc. of the ARPA Image Understanding Workshop 1994, Monterey, CA, 1994

5. Hartley, R.: Invariants of points seen in multiple images. Technical Report, G.E. CRD, Schenectady, 1992

6. Hartley, R., Gupta, R., Chang, T.: Stereo from uncalibrated cameras. Proc. of the Conf. on Computer Vision and Pattern Recognition, Urbana-Champaign, Illinois, USA, 1992, 761-764

7. Luong, Q.T., Vieville, T.: Canonic Representations for the Geometries of Multiple Projective Views. Technical Report, University of California, Berkeley, 1993

8. Mohr, R., Quan, L., Veillon, F.: Relative 3D reconstruction using multiple uncalibrated images. The International Journal of Robotics Research, 1995, to appear 
9. Quan, L.: Affine stereo calibration for relative affine shape reconstruction. Proc. of the 4th British Machine Vision Conference, Surrey, England 1993, 659-668

10. Sturm, P.: Affine Stereo Calibration. Technical Report, LIFIA, No. 29, 1995 